\title{
On probing human fingertips in vivo using near-infrared light: model calculations
}

\author{
Joseph Chaiken \\ Jerry Goodisman \\ Syracuse University \\ Department of Chemistry \\ and \\ Department of Biomedical Engineering \\ Syracuse, New York 13244-4100
}

\begin{abstract}
We probe volar-side fingertip capillary beds with nearinfrared laser light and collect Raman, Rayleigh, and Mie scattered light and fluorescence. The results are interpreted using radiation transfer theory in the single-scattering approximation. The surface topography of the skin is modeled using the Fresnel equations. The skin is treated as a three-layer material, with a mean-field treatment of tissue composition and related optical properties. The model, with a reasonable choice of tissue parameters, gives a remarkably accurate account of the features of actual measurements. It predicts the optimal values for the incident angle of the laser beam and the distance between beam and detector. It explains the correlated temporal changes in the intensities of elastically and inelastically scattered light caused by heart-driven pulses and why they are out of phase. With appropriate boundary conditions, the model can be used to discuss the scattering from ridged skin extruded conformally into an aperture in a metal surface under constant light pressure. The probing results suggest an inherent regularity and similarity in the anatomy and composition of surface and subsurface tissues of a wide range of skin types. (C) 2010 Society of Photo-Optical Instrumentation Engineers. [DOI: 10.1117/1.3431119]
\end{abstract}

Keywords: Raman; in vivo; blood analysis; glucose; noninvasive.

Paper 09546RR received Dec. 9, 2009; revised manuscript received Mar. 29, 2010; accepted for publication Apr. 1, 2010; published online Jun. 1, 2010.

\section{Introduction}

In order to measure blood and tissue analytes noninvasively in vivo, we probe volar-side fingertips with near-infrared radiation (NIR). We simultaneously measure the elastically scattered light and the undifferentiated inelastically scattered light, containing both Raman scattered light and fluorescence. Whereas vibrational spectroscopy-specifically, Raman scattering with NIR excitation, ${ }^{1,2}$ - provides chemically specific and quantitative information concerning molecules in complex mixtures such as blood ${ }^{3}$ in vivo, the elastically scattered light, a probe based on physical optics, provides information relating to the presence and disposition of red blood cells (RBCs) in the probed volume.

In the present paper, we present and discuss our model for analysis and interpretation of our measurements. The model considers the scattering and absorption, inside the skin, of a beam of NIR, and the propagation of the scattered radiation to a detector outside. (We assume that the scattering is isotropic-i.e., no preferred scattering angle.) It is used to discuss the effects of skin surface topography-i.e., doming and ridges - as well as the layer structure of skin. This is in the context of an earlier study ${ }^{4,5}$ in which we analyzed the sources of variation in glucose concentrations obtained from in vivo Raman spectroscopy of volar-side fingertip capillaries.

Address all correspondence to Joseph Chaiken, Syracuse University, Center for Science and Technology, Department of Chemistry, Syracuse, New York 13244 4100. Tel: 315-443-2925; Fax: 315-443-4070; E-mail: jchaiken@syr.edu
Ridged skin ${ }^{6,7}$ such as is found on volar-side fingertips provides an important thermoregulation function and so is highly vascularized, presenting a blood-rich tissue to probe. However, the surface ridges can be commensurate with the diameter of the laser beam, making results depend on the placement of the laser beam on the skin surface. The sample being measured-e.g., the tissue-must be brought into reproducible registration with an optical system. In our apparatus, ${ }^{8}$ an external servo-driven actuator presses the fingertip against a circular aperture in a thin spring steel sheet, and an NIR laser beam penetrates the skin through the exposed surface. The various forms of emitted light are measured simultaneously with a CCD detector.

The blood transmits a local pressure field into the probed volume, the diastolic and systolic pressure, in addition to the local hydrostatic and oncotic pressures. It is a fact ${ }^{8-10}$ that pressure on the fingertip, even very light constant pressure, has several effects. To the degree that the skin can deform, ${ }^{11}$ the pressure causes "doming" or extrusion of tissues into an aperture. Second, over time, constant pressure results in movement of fluid, e.g., blood, out of the irradiated region. Also, as fluid moves in response to the applied pressure, the contact area between the tissue and the surface containing the aperture changes, requiring an external servo system to obtain reproducible results.

Regardless of how the subsurface propagation is calculated, the amounts of inelastically scattered light and fluorescence collected are proportional to the intensity of incident

$1083-3668 / 2010 / 15(3) / 037007 / 15 / \$ 25.00$ @ 2010 SPIE 
light. The Fresnel equations are appropriate for calculating the effect of spatial inhomogeneity on light propagation when the path contains features (such as fingerprint ridges), which are large compared with near-infrared (NIR) wavelengths. The scattering coefficients ${ }^{12}$ account for interactions with interfaces and objects having index of refraction boundaries that are equal to or smaller than the NIR wavelength. Given the well-defined anatomy of highly organized skin tissues, in vivo probing encounters a multitude of randomly arranged refractive index boundaries that are properly represented by the tabulated scattering coefficients.

Our goal is to construct a model that provides a conceptual framework that accounts for all the experimental observations and if desired could allow a best fit to quantitative measurements by adjusting a small number of parameters. Although it is possible to conceive of detailed models of skin structure with as many as five layers and many parameters, ${ }^{13}$ the possibilities for obtaining enough observables to properly test such a model are limited. Zhang ${ }^{12}$ suggests that a three-layer model is reasonable in light of available empirical data, and we use such a model. The three layers, designated as a, b, and $\mathrm{c}$ from top to bottom, are the stratum corneum and the superficial epidermis, the "capillary bed" that is mostly epidermis but extends inward to the superficial dermal plexus, and the dermis, respectively. We consider first a planar geometry (in the model, layers $a$ and $b$ are defined by parallel planes) and calculate the dependence of measured intensities on experimental geometric parameters. We then consider the effect of extrusion into the aperture of the uppermost layer-i.e., "doming" of the top surface of layer a. We also calculate the effect on scattered intensities of known changes like net blood flow into/out of the probed volume. Last, we include the effect of fingerprint ridges, which we assume differs from the effect of subsurface scattering.

The optical properties of the constituents of vascularized skin-i.e., plasma, RBCs, dermis, and epidermis - have been measured many times, giving mean-field values for the region probed. Jacques ${ }^{14}$ has estimated that in the most vascularized "layer," the superficial dermal plexus 200 to $300 \mu \mathrm{m}$ below the skin surface, the blood (plasma and RBCs) volume percent is 3 to $5 \%$. Using independently measured scattering and absorption coefficients, we can explicitly calculate only the elastic scattering (EE), but as we can calculate the path of the incoming light, in principle it is possible to calculate the Raman and fluorescence (IE) produced. To calculate Raman and fluorescence emission in our experiments, we require the relevant Raman cross sections, ${ }^{2}$ volume percents for all phases giving distinct Raman signals, and quantum yields for fluorescence, in addition to the absorption coefficents that we already possess. In principle, these parameters relate the model we present to chemometric analyses ${ }^{15,16}$ of in vivo Raman spectra and those of turbid media.

Perhaps the most important approximation in our model is the neglect of multiple scattering, so we address this immediately. Although appreciable fluorescence emanates from all the tissues in the probed volume, the RBCs are the strongest source, followed by the plasma and then the static tissues. An estimate $^{17}$ of the volume fraction of blood in the probed volume is $5 \%$. In clinical practice, ${ }^{18}$ the measured hematocrit (volume fraction of blood that is RBCs) depends on the size of the vessel from which the blood is sampled. The hematocrit

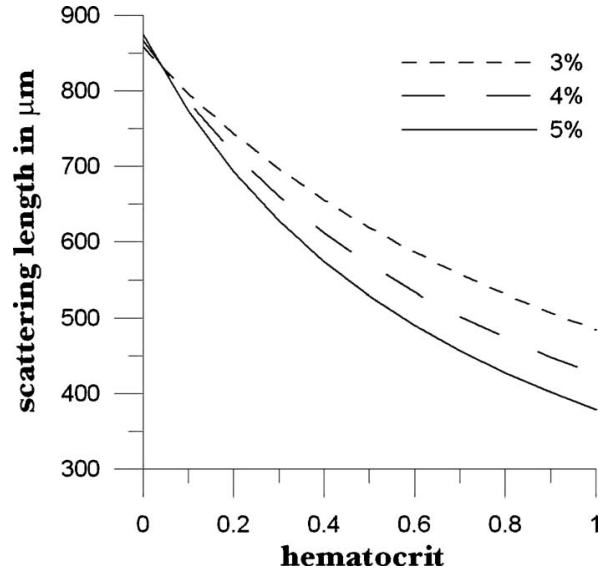

Fig. 1 Calculated scattering length of 830-nm radiation in medium having total blood volume of 3 to $5 \%$ and hematocrit shown on abscissa, with remainder of volume filled with epidermis. Scattering coefficients are taken from Refs. 12 and 13.

of blood from a large vessel like the brachial vein is about $45 \%$, but the hematocrit of capillary blood is about $10 \%$ or less on average. Using the scattering coefficients tabulated in Table $2,{ }^{19,20}$ one can calculate the scattering length of photons in skin for a range of total blood volumes and hematocrits, with the results shown in Fig. 1. Scattering lengths are almost always much larger than $300 \mu \mathrm{m}$. Since the capillary bed itself is only about $200 \mu \mathrm{m}$ thick, it is clear that a singlescattering model of light propagation should suffice. The scattering length in all the other tissues is even longer than in the capillary bed.

Sufficient detail to allow reproduction of our results has been published ${ }^{3,4,8,21}$ elsewhere, so our intent in the next section is to provide sufficient detail to allow comparison of our modeling results with the practical and experimental facts. We then present some distinctive experimental observations associated with probing volar-side fingertip capillary beds, followed by a presentation of the model. Ultimately, we compare the calculated results with the experimental data in order to demonstrate the validity of this model and the assumptions.

\section{Experiment}

The volar side of a fingertip is placed in contact with a rigid, flat, stiff (0.025-in-thick spring steel) surface having a $\approx 2$-mm hole, chamfered $(10 \mathrm{deg})$ on the side facing the incident light. The incident light is free-space coupled to the skin with a $15-\mathrm{cm}$ focal length plano convex lens. The size of this hole and the chamfering are chosen to minimize extrusion of tissue and to minimize the scattered light produced by interaction of the laser with the edges of the hole. The hole and surrounding surface cause a pressure-dependent stress field within the tissues in direct and indirect contact with the edges of the hole, which leads to motion of subsurface tissues.

Data are obtained using highly polarized, external cavity diode lasers (depending on specific purposes, we use either Process Instruments, Salt Lake, Utah, or Sacher Lasertechnik Tiger Model, Marburg, Germany) with clean-up and edge filters (Semrock, Rochester, New York). The following data shown correspond to $830-\mathrm{nm}$ and $200-\mathrm{mW}$ excitation. Be- 
cause we propagate the laser light with an angle of incidence of $53 \pm 1 \mathrm{deg}$ (the uncertainty is the practical limit and not a precisely known number) to the normal to the plane of the aperture, we observe the laser cross section on the finger to be elliptical. Using knife-edge scanning experiments and Zap-it paper, we estimate the elliptical spot to have a $100-\mu \mathrm{m}$ minor axis and a 237- $\mu \mathrm{m}$ major axis. The spectrograph (Process Instruments) is fiber-coupled to a collection train consisting of a custom triplet collection lens, followed by a (Semrock Razor Edge) filter to remove the laser line, and then a refocusing lens. The 59-fiber bundle presents an AR-coated circular target to the refocusing lens and a line configuration at the spectrograph entrance slit. The effective slit width is $70 \mu \mathrm{m}$, and the net collection and spectrograph system is approximately $f / 2$. The wavelength-dispersed light is imaged onto either an Andor DU420-BR-DD CCD camera operating at $-65{ }^{\circ} \mathrm{C}$ or a Critical Link MityCCD-E3011-BI CCD camera, cooled to $-65^{\circ} \mathrm{C}$.

In order to view the skin surface through the modulating aperture in real time, a $5 \%$ reflectance pellicle can be introduced at 45 deg between the custom triplet and the refocusing lens. Illumination can be provided by a white-light source from the collection side. The aperture (finger) can be imaged in real time through the triplet directly and reflected onto a separate NIR-enhanced CCD camera. Images were captured using a Snappy frame grabber. White-light illumination easily saturates the viewing CCD camera. On the other hand, under even low-power laser illumination, e.g., $25 \mathrm{~mW}$, the dynamic range of the CCD is challenged in attempting to use the Raleigh/Mie scattered light for spectral probing and imaging. Therefore, judicious use of neutral density filters is required to deal with illumination levels and saturation blooming to obtain good images.

We refer to the pressure-induced motion as tissue modulation. In order to reproducibly execute these and even static pressure experiments, one must utilize a system that allows accurate and precise placement and pressure maintenance, as we have described elsewhere. ${ }^{4,8,21}$ It is also possible to employ wavelength-selective filters ${ }^{22}$ to image the skin and thereby deduce perfusion patterns, but for our current purposes, we find scanning the laser across the aperture while simultaneously collecting both the IE and the EE to be more useful. For this, the entire assembly containing the aperture is mounted on a stage that can be driven electromechanically relative to the stationary laser and collection system using a Newport NSA12 micropositioner.

In order to understand the behaviors of the IE and the EE, we examine the background produced by the specular reflection off the skin surface, which is indistinguishable from the EE scattered background. To characterize how the collection system records specular reflection from the probed skin in the absence of any subsurface or any IE contribution, we performed experiments using stainless steel ball bearings of various diameters in place of the finger in the optical aperture. We have previously described ${ }^{21}$ some results of experiments using ball bearings that relate directly to the production of highly off axis light in the collection system, since this can also contribute to the apparent background at all Raman shifts. The ball bearings must be new and clean, and saturation of the CCD must be avoided, perhaps using an additional laser re-

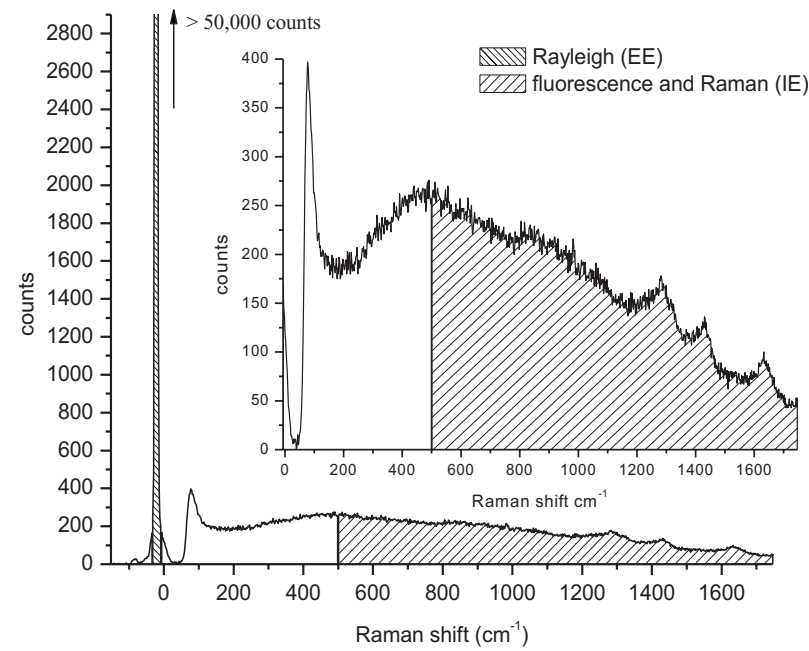

Fig. 2 Intensity versus frequency from a typical 20-ms frame of Andor CCD output with system optimally aligned using 200-mW and 830-nm excitation. The sections used to calculate inelastic scattering intensity IE $\left(\approx 500\right.$ to $\left.1750 \mathrm{~cm}^{-1}\right)$ and elastic scattering intensity EE $\left(-30 \mp 10 \mathrm{~cm}^{-1}\right)$ are shown. The low shift integration limit for obtaining IE was chosen by reference to the output obtained when the sample is a nonfluorescent metal and requiring that no EE is included in the IR integral.

jection filter, to avoid measurement artifacts and to prevent damage to the CCD array.

\section{Experimental Results}

Measured scattered intensity as a function of wavelength, from a single CCD frame, not corrected for instrument response, is shown in Fig. 2. The regions used to calculate the integrated inelastic (IE) signal and the integrated elastically emitted (EE) radiation are indicated. The EE is dominated by elastically scattered light from all the tissues in the irradiated volume, but it also contains light reflected from the skin surface. The inelastic signal includes fluorescence and Raman scattered light. The IE is necessarily much smaller than the EE.

The optical system is initially aligned so that the light is incident at the middle of the aperture if the sample is flat and in the plane of the aperture, as can be seen in Fig. 3(a) for a thick flat piece of light gray Delrin. When a finger is registered into the aperture in an unpressed state, as in Fig. 3(b), the ellipse of contact is slightly shifted to the side of the tissue toward the incoming laser light. If additional pressure is applied, the point of contact moves farther toward the side of the incoming laser, as in Fig. 3(c). This motion, as we discuss in detail in the following, is down the side of the extruded dome of tissue. Figure 3(d) shows the difference between images $b$ and $\mathrm{c}$ and demonstrates that the total displacement, even for a very hard applied pressure, is only 10 to $30 \mu \mathrm{m}$.

In order to probe skin topography, we translate the skin relative to the laser beam, either by moving the finger relative to the aperture with the aperture stationary relative to the excitation and collection optics, or by moving the aperture relative to the excitation and collection optics with the skin stationary relative to the aperture. In the latter case, the amount of off-axis elastic light production depends on the location of 


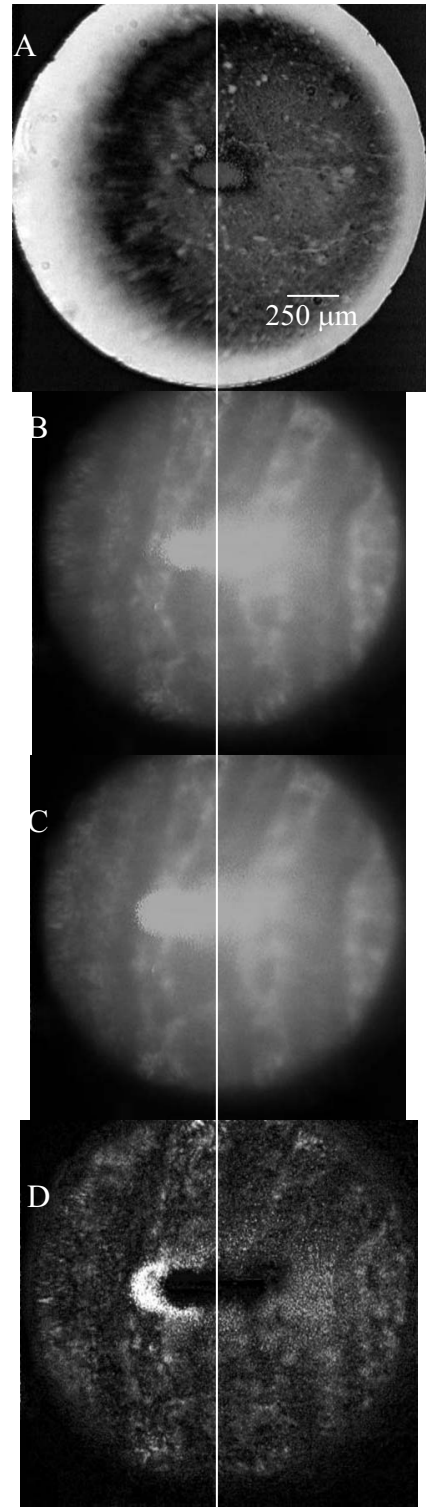

Fig. 3 (a) Backlit flat Delrin block. (b) Unpressed fingertip (pressure $\sim 45 \mathrm{~g} / \mathrm{cm}^{2}$ ), showing illumination at center. (c) Fingertip pressed hard (pressure $>140 \mathrm{~g} / \mathrm{cm}^{2}$ ), showing displacement of illuminated area. (d) Difference between pressed (c) and unpressed (b).

the edges of the aperture relative to the laser. Figure 4 shows data obtained by the former approach. Here, we scanned a part of the fingertip where the ridges run perpendicular to the direction of motion, the fingertip being moved through a known distance in a known amount of time with relatively constant applied pressure. ${ }^{8,2}$ The IE and EE integrals shown in Fig. 2 are plotted versus distance translated. Both fluctuate by roughly $20 \%$ with a spacing of about $250 \mu \mathrm{m}$, characteristic of fingerprint ridges. Interestingly, the spatial fluctuations in the EE integral and the IE integral are complementary: one increases when the other decreases.

Similar out-of-phase oscillations are observed in pulsemodulated time-dependent measurements, ${ }^{5}$ in which the pulse causes a periodic increase in $\mathrm{RBC}$ volume fraction. Increased volume fraction of RBCs necessarily causes an increase in the IE, but this is accompanied by a decrease in the EE. We will

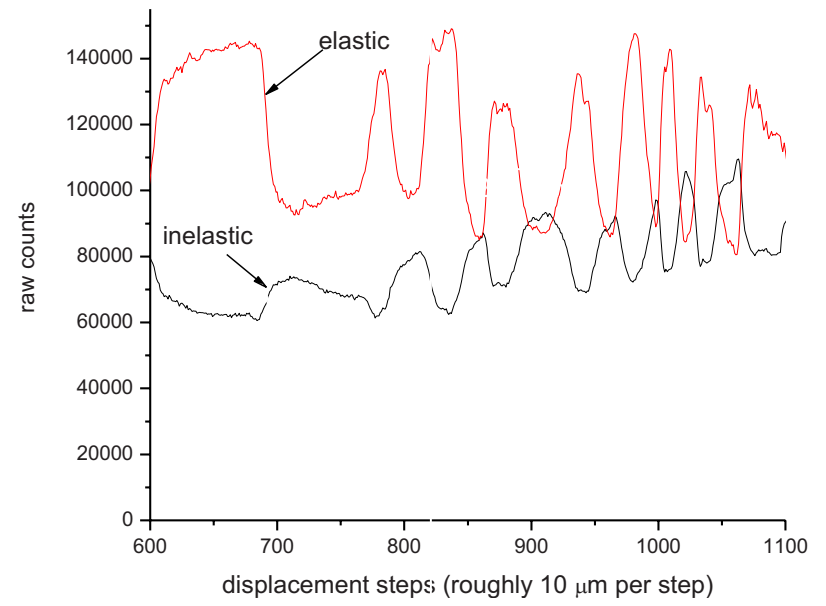

Fig. 4 EE (elastic) and IE (inelastic) scattered light integrals from scan of laser and detector across fingerprint ridges plotted against displacement of fingertip alone across a stationary aperture.

show in the following that although the explanations are different, the temporal fluctuations due to heart pulsing and the spatial fluctuations caused by fingerprint ridges are both predicted by our model. As we have noted, if the aperture is large enough to include a few fingerprint ridges, extrusion of the fingertip into the aperture-i.e., doming-occurs.

With our apparatus, a skilled test subject can scan his/her finger across the aperture, with known pressure and acceptable spatial precision and accuracy, using visual feedback from the apparatus. This involvement of the test subject limits the reproducibility, precision, and accuracy of the results. Therefore, most of the data presented were obtained using the second approach, in which the fingertip moves with the moving aperture. Direct observation through the collection system shows that nearly all test subjects can remain stationary to within $\pm 10 \mu \mathrm{m}$ relative to the aperture for hundreds of seconds, in spite of inevitable involuntary tremors and similar random motions.

Figure 5 shows data obtained using the second approach for four test subjects: a petite Asian female (28 years old), a large Caucasian male (19 years old), an average-size Caucasian male (54 years old), and an average-size Middle Eastern male (78 years old). In each case, the pressure used to maintain optical registration between the fingertip and the aperture was the average of the diastolic and systolic pressures measured with a commercial automatic blood pressure cuff (Omron). The data consists of 50 consecutive 20-ms CCD frames acquired at fixed positions spaced at $20-\mu \mathrm{m}$ intervals. A large increase in $\mathrm{EE}$ at the extreme right due to reflection as the laser beam strikes the outside of the aperture has been cropped. Oscillations apparently due to fingerprint ridges are evident, but so is a steady decrease or increase in intensity as one goes from left to right. As shown in the following, this is associated with the doming of the fingertip pressed on the aperture. Careful experimentation revealed that for stainless steel ball bearings having curvature similar to fingertips, some reflected light from the bearings propagates through the entrance aperture of the first collection triplet. The smaller the ball, the larger the height of the simulated dome and the smaller the displacement of the aperture needed to scan the 

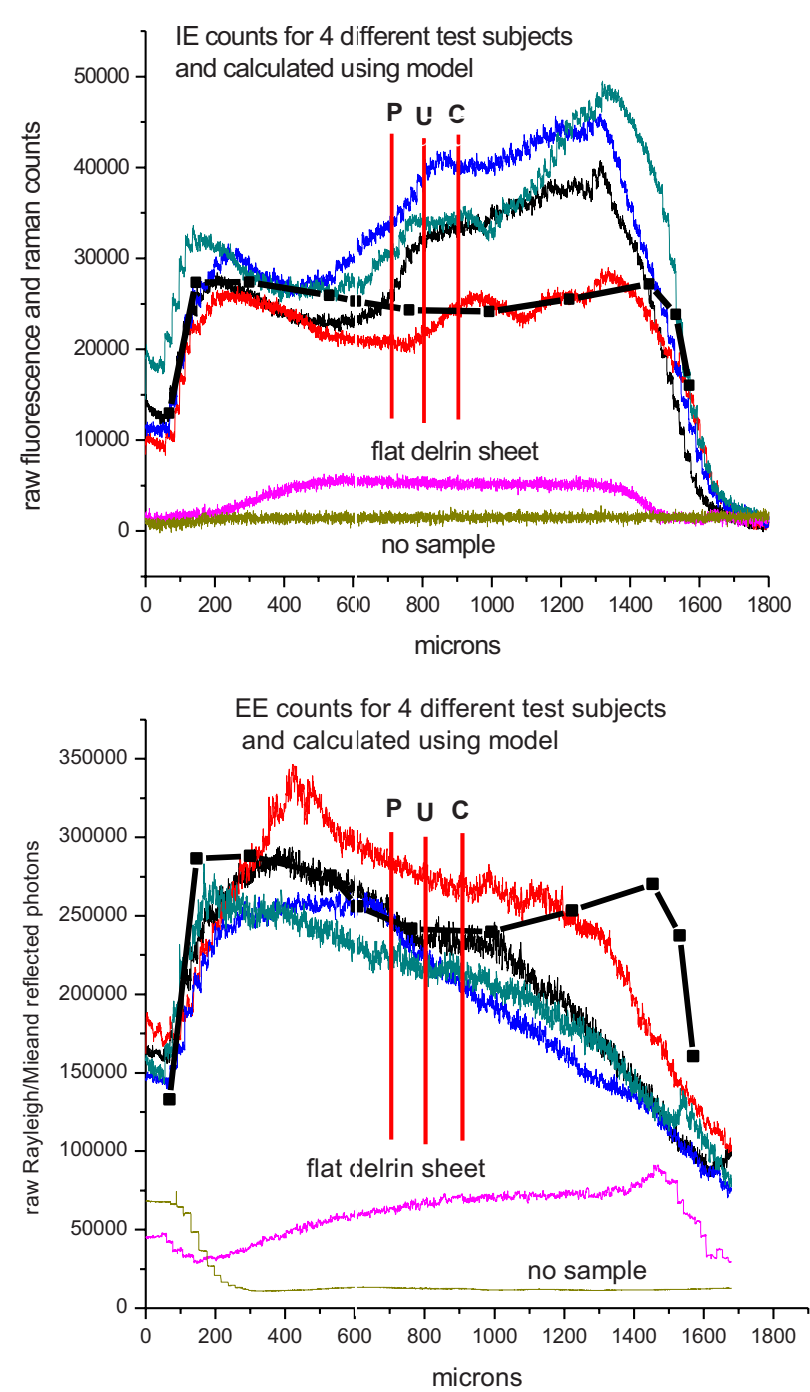

Fig. 5 Elastic and inelastic scattering integrals (EE and IE) for four human subjects, plotted versus displacement of the fingertip and aperture relative to the laser beam and the detector (fingertip stationary with respect to the aperture; laser beam stationary with respect to detector). Also shown are the EE and IE for a flat Delrin sheet and for a blank (no sample). The calculated EE includes specularly reflected light that increases when the reflected beam scans across the aperture with changing $x_{s}$. The calculated IE is the EE result without the specular reflection contribution. Results of calculations described in the text are indicated by thick black lines joining black squares. The positions of the laser entrance point at the different times of a tissue modulation sequence are shown at $\mathrm{U}, \mathrm{C}$, and $\mathrm{P}$, as described in the text.

reflected light across the entire collection system entrance aperture.

\section{Model Used for Calculations}

In our "simple" model, a beam of NIR laser light is incident on the top surface of skin at an angle $\theta^{\prime}$ relative to the vertical, as shown in Fig. 6, which assumes planar geometry-i.e., smooth skin with no extrusion into the aperture. The apparatus is aligned so that the angle of incidence of the light-i.e., $\theta^{\prime}$ - is near Brewster's angle for an index of refraction for layer a equal to that of water. The actual index of refraction of "tissue" is somewhat higher, but observations reflect modula-

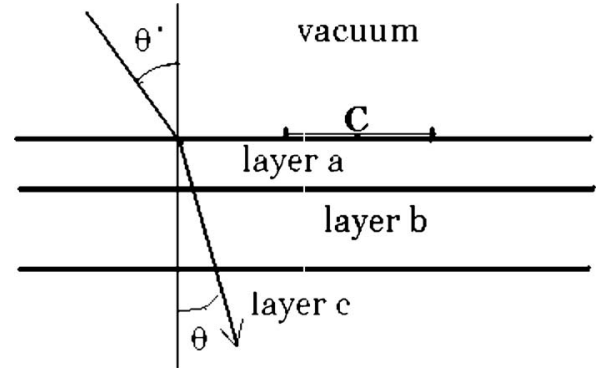

Fig. 6 Geometry of the experiment: Radiation is incident on the skin, modeled as three planar layers. The incident beam makes an angle $\theta^{\prime}$ with the outward normal, which is changed to $\theta$ inside the skin by Snell's law refraction.

tion of the optical signals due to the motions of subsurface fluids and deformation of static tissues, which do not reflect exactly the anatomical divisions for which individual indices have been measured-i.e., stratum corneum, epidermis, dermis. Thus, we use water for reference.

In this case, most of the radiation penetrates (because the angle of incidence is constant, the transmission coefficient is constant for any point of entry and is close to unity), but light reflected to the detector is not negligible, particularly when the EE is being observed. We estimate the amount of light reflected from the skin as a function of the relative position of the incoming laser and the aperture in a separate calculation. Inside the skin, the radiation is attenuated by scattering and absorption, with the scattering assumed to be spherically symmetrical. This assumption has been used by others ${ }^{23}$ and seems reasonable in our case, given the known interdigitation ${ }^{6,7}$ of tissues at subsurface layer boundaries with size large compared with the incident and scattered light wavelengths.

Assuming planar geometry, some of the scattered radiation is propagated upward and crosses the flat skin-air interface, where it is refracted and eventually is collected and detected. We represent the detector as a circular area parallel to the skin surface, with its center, $\mathrm{C}$ in Fig. 6, a distance $d$ from the center of the incoming laser beam. The diameter is consistent with the net $f$-number of the first collection lens. Radiation passing through this "collection circle" from below is considered detected. We calculate the amount of radiation entering the collection circle, given the intensity and direction of incoming radiation and the parameters characterizing the skin. The main assumption made is single scattering-i.e., each photon entering the detector is produced by a single scattering event.

To introduce the effect of doming into the model-i.e., the observed extrusion of tissue into the aperture-the planar interface is replaced by a spherical cap. In this case, the incoming or outgoing transmission coefficient depends on the points at which incoming or outgoing radiation enters and leaves the skin. In either planar or dome geometry, radiation passing through this collection circle from below is considered detected.

The subsurface skin is modeled as a three-layer planar system. The top layer (layer a, stratum corneum plus the upper nonviable part of epidermis starting at the distal ends of the capillaries) has thickness $t_{a}$, the middle layer (layer b, epider- 
Table 1 Volume fractions of three tissue phases in skin.

\begin{tabular}{lccc}
\hline Phase & Layer $a$ & Layer $b$ & Layer $c$ \\
\hline$p=$ plasma & 0.00 & 0.0072 & 0.001200 \\
$r=$ red blood cells & 0.00 & 0.0008 & 0.000133 \\
$t=$ static tissue & 1.00 & 0.9920 & 0.998667 \\
\hline
\end{tabular}

mis including capillary bed ending with projection of vessel to superficial dermal plexus) has thickness $t_{b}$, and the third layer (layer c, dermis) is assumed to be infinitely thick. The last assumption is not a source of error because only a small fraction of the collected light comes from layer $\mathrm{c}$, due to the attenuation of both the incident and the scattered light. Typically, we take $t_{a}=0.02 \mathrm{~cm}$ and $t_{b}=0.04 \mathrm{~cm}$. Three phases are present: $r=$ red blood cells, $p=$ plasma, and $t=$ static tissue. The volume fractions of the three phases in the three layers are denoted by $\phi_{r a}, \phi_{p a}, \phi_{t a}, \phi_{r b}$, etc. Of course, $\phi_{r a}+\phi_{p a}$ $+\phi_{t a}=1$ and so on. The volume fractions used typically in our calculations are given in Table 1.

Each phase has characteristic absorption and scattering coefficients, denoted by $\alpha$ and $\mu$, respectively. Typically, we use the values shown in Table 2. For our purposes, we can assume that the phases are distributed randomly in each layer. This allows us to calculate an average absorption coefficient and an average scattering coefficient for each layer and a total attenuation coefficient that is the sum of the absorption and scattering coefficients. For instance, $\alpha_{b}=\phi_{r b} \alpha_{r}+\phi_{p b} \alpha_{a}+\phi_{t b} \alpha_{t}$ and $\mu_{c}=\phi_{r c} \mu_{r}+\phi_{p c} \mu_{p}+\phi_{t c} \mu_{t}$.

The incident radiation is assumed to enter the skin from the space above as a beam centered at the origin, $\mathrm{O}$, and making an angle $\theta^{\prime}$ with the normal to the skin layers (Fig. 6). Inside the skin, the angle between the normal and the direction of propagation is $\theta$, where $\theta$ is related to the external angle $\theta^{\prime}$ by Snell's law, $\sin (\theta)=\sin \left(\theta^{\prime}\right) / n_{s}$ assuming index of refraction $n_{s}=1.3$ in the skin. Since all three layers have about the same value of $n$, the beam is assumed to undergo no further change of direction inside the skin. The transmission coefficient for radiation ${ }^{24}$ entering the top layer is calculated from the reflection coefficients for $\mathrm{s}$ - and $\mathrm{p}$-polarized radiation,

$$
R_{s}=\left[\frac{\cos \left(\theta^{\prime}\right)-n_{r} \cos (\theta)}{\cos \left(\theta^{\prime}\right)+n_{r} \cos (\theta)}\right]^{2} \quad R_{p}=\left[\frac{\cos (\theta)-n_{r} \cos \left(\theta^{\prime}\right)}{\cos (\theta)+n_{r} \cos \left(\theta^{\prime}\right)}\right]^{2} .
$$

The transmission coefficient $T$ is $1-\left(R_{s}+R_{p}\right) / 2$. With $n_{r}$ $=1.3$ and $\theta^{\prime}=0.63$, we get $\theta=0.47$ and $T=0.981$. We use the Fresnel equations as if the light were unpolarized to attempt to simulate three-dimensional variation in the skin surface, since to the extent that there is three-dimensional tortuousity, both polarizations would contribute to the reflected light and the reflection loss for penetration into the skin. We make this assumption to justify our choice to calculate the trajectory at the interface in two dimensions only. Although the reflected light is only one to two percent of the incident light, its intensity is much greater than the elastically scattered light from
Table 2 Absorption and scattering coefficients for three phases.

\begin{tabular}{lcc}
\hline \multicolumn{1}{c}{ Phase } & Absorption coefficient & Scattering coefficient \\
\hline$r=$ RBCs & $\alpha_{r}=4.5 \mathrm{~cm}^{-1}$ & $\mu_{r}=300 \mathrm{~cm}^{-1}$ \\
$p=$ plasma & $\alpha_{p}=0.3 \mathrm{~cm}^{-1}$ & $\mu_{p}=0.6 \mathrm{~cm}^{-1}$ \\
$t=$ static tissue & $\alpha_{t}=5 \mathrm{~cm}^{-1}$ & $\mu_{t}=12 \mathrm{~cm}^{-1}$ \\
\hline
\end{tabular}

plasma and RBCs (see the following); it is much smaller than that from static tissue.

As shown in Fig. 7(a), we take the vertical axis as the $z$ axis, the positive direction being down-i.e., into the skin. The incident beam is in the $x-z$ plane. The direction of propagation of the incident beam in the skin is shown in the figure by the line OP and its continuation. If $\mathrm{B}$ is the $x$ value of point $\mathrm{P}, \sin \theta=B / l$. We also define the angle $\alpha$ as $(\pi / 2)-\theta$.

We first calculate the intensity of the beam at each point along its trajectory. If $I_{o}$ is the beam intensity for $z<0$ and $l$ is the length of the beam within the skin, the beam intensity at point $\mathrm{P}$ is

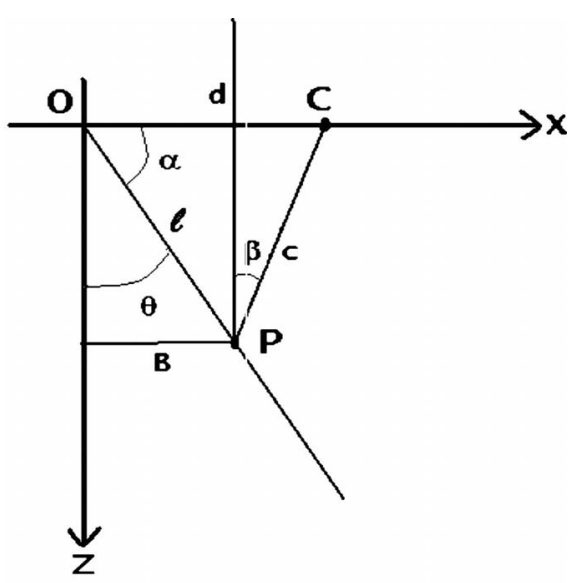

(a)

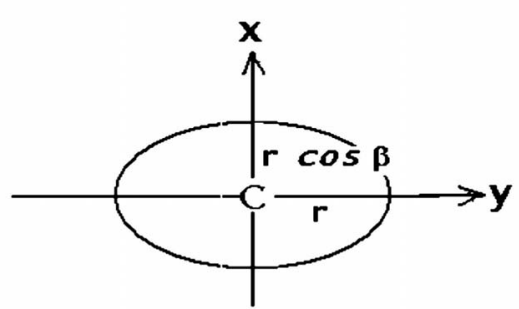

(b)

Fig. 7 (a) Geometry of the experiment inside the skin: $z=0$ is the top boundary of layer $\mathrm{a}$, and the beam inside the skin makes an angle $\theta$ with the $z$ axis. The center of the beam enters the skin at the origin $O$ and propagates a length / to P, where scattering occurs. (b) Top view, showing the projection of the collection circle in a plane perpendicular to PC. 


$$
I=I_{o} \exp \left[-\int_{0}^{l}(\alpha+\mu) \mathrm{d} t\right]
$$

Here, $\alpha+\mu$ is the attenuation coefficient, which depends on $z=t \cos (\theta)$ because $\alpha$ and $\mu$ are different in layers a, b, and c. The probability of scattering by phase $x(x=r, p$, or $t)$ at the point $\mathrm{P}$ is proportional to the scattering coefficient $\mu_{x}$ and to the volume fraction of phase $x$ in the layer in which point $\mathrm{P}$ is located. The scattering probability must be multiplied by the probability that the scattered radiation enters the collection circle, $\mathrm{P}_{\mathrm{c}}$.

Since scattering is assumed isotropic, $\mathrm{P}_{c}$ is equal to the ratio of the solid angle subtended by the collection circle to $4 \pi$. To calculate this solid angle, note that in the plane passing through point $\mathrm{C}$ and perpendicular to the line $\mathrm{PC}$, the collection circle projects to an ellipse with semiaxes $r$ (in the $y$ direction) and $r /(\cos \beta)$ (in the direction perpendicular to $y$ ), where $\beta$ is the angle between the line PC and the $z$ direction [see Fig. 6(b)]. The solid angle subtended by the ellipse is the solid angle we seek. Consider a system of polar coordinates with PC being the polar axis, $\theta$ the polar angle, and $\varphi$ the azimuthal angle, and let $x^{\prime}, y^{\prime}$, and $z^{\prime}$ be the Cartesian axes with $y^{\prime} \equiv y$ and $z^{\prime}$ along PC. Let $\rho$ be the distance from $\mathrm{P}$ to a point on the ellipse so that $x^{\prime}=\rho \cos \varphi$ and $y^{\prime}$ $=\rho \sin \varphi$. The maximum value of the polar angle is $\tan ^{-1}(\rho / c)$. From the equation of the ellipse,

$$
\frac{\left(x^{\prime}\right)^{2}}{\cos ^{2} \beta}+\left(y^{\prime}\right)^{2}=r^{2},
$$

we obtain

$$
\rho=\frac{r}{\left(\frac{\cos ^{2} \varphi}{\cos ^{2} \beta}+\sin ^{2} \varphi\right)^{1 / 2}}
$$

Thus, the solid angle subtended by the ellipse is

$$
\int_{0}^{2 \pi} \mathrm{d} \varphi \int_{0}^{\tan ^{-1}(\rho / c)} \sin \theta \mathrm{d} \theta=\int_{0}^{2 \pi} \mathrm{d} \varphi\left\{1-\frac{1}{\left[1+(\rho / c)^{2}\right]^{1 / 2}}\right\} .
$$

Substituting for $\rho$ and dividing by $4 \pi$, we find the probability that radiation scattered isotropically from point $\mathrm{P}$ enters the collection circle to be

$$
\mathrm{P}_{\mathrm{c}}=\frac{1}{2}-\frac{c}{\pi} \int_{0}^{\pi / 2} \mathrm{~d} \varphi\left[c^{2}+\frac{r^{2}}{\frac{\cos ^{2} \varphi}{\cos ^{2} \beta}+\sin ^{2} \varphi}\right]^{-1 / 2}
$$

This factor multiplies the intensity of scattering from the point P.

We must also consider the attenuation of the scattered radiation on its way to the detector. This factor may be written as $\exp \left[-\int_{0}^{m}(\alpha+\mu) \mathrm{d} t\right]$, where $m$ is the length of the line PC, and $\alpha+\mu$ depends on $z=t \cos \beta$ because the attenuation coefficient is different in each layer. Taking all of the preceding into account, we write the total detected radiation scattered from phase $x$ as

$$
\begin{aligned}
I_{n e t}= & I_{o} T \int_{0}^{\infty} \mathrm{d} l \exp \left[-\int_{0}^{l} \mathrm{~d} t(\alpha+\mu)\right] \times \mu_{x} \phi_{x}(z) \times \mathrm{P}_{\mathrm{c}} \\
& \times \exp \left[-\int_{0}^{m} \mathrm{~d} t(\alpha+\mu)\right],
\end{aligned}
$$

where $z=l \cos \theta$ in the first integral over $t$, and $z=t \cos \beta$ in the second integral over $t$. The integral over $l$ sums contributions from all points along the line OP (extended to a depth so that further contributions are negligible).

The quantity $I_{n e t}$ represents the scattering from an infinitely narrow beam. To account for the circular cross section of the beam, we calculate $I_{n e t}$ for four entry points along the $x$ direction and average. That is, if $d$ denotes the entry point for the center of the beam, and $d b$ the beam diameter (here, $0.01 \mathrm{~cm}$ ), we repeat the calculation for $d-0.40451 d b, d$ $-0.15451 d b, d+0.15451 d b$, and $d+0.40451 d b$ and multiply the four results by $0.13820,0.31680,0.31680$, and 0.13820 , respectively. These are the four-point Gaussian quadrature points and weights ${ }^{25}$ for integrating over a circular area. The sum of the four terms is the scattered intensity averaged over a circle of diameter $d b$.

\section{Predictions of Geometrical Parameters}

Calculations using this model were used to establish the optimal values of geometrical parameters of the experimental apparatus. For all calculations, we use the volume fractions and other parameters given in the tables. We can calculate collected intensities for scattering from each of the three phases, and these will be shown. In an actual experiment, one can distinguish between scattering from different phases by Raman shifts and the behavior of the signals under modulation. In any case, the IE is dominated by scattering (fluorescence) from RBCs.

We first determine the optimum angle $\theta^{\prime}$ for a fixed value of $d$, the distance between the center of the beam and the center of the collection circle. Corresponding to the actual geometry of our apparatus, the results presented here are for $d=0.014 \mathrm{~cm}$, diameter of the collection circle $=0.01 \mathrm{~cm}$, and diameter of the input beam $=0.01 \mathrm{~cm}$. We vary the angle $\theta^{\prime}$ between the beam axis and the normal to the outside of the top skin layer and calculate the collected scattered intensities for each of the three phases, adding together intensities for all three layers.

The results are shown in Fig. 8. From top to bottom, the curves correspond to static tissue, red blood cells, and plasma. For clarity, we have drawn polynomial fits (cubic, quadratic, and cubic, respectively). The maxima in the three plots, calculated from the data itself, are at $\theta^{\prime}=1.192,0.677$, and 0.677 , respectively. Since the scattering from static tissue is dominated by the contribution of the top layer, the optimum beam direction is farther from the normal. For blood and plasma scattering, a beam direction closer to the normal gives a deeper penetration and more signal-i.e., more of layers $b$ and $\mathrm{c}$ - for a fixed distance between beam and detector.

We conducted calculations in which the value of $\theta^{\prime}$ is fixed and the value of $d$ (distance between the center of the beam and the center of the collection circle) was varied. We calculated the detected scattered light originating from static tissue, 


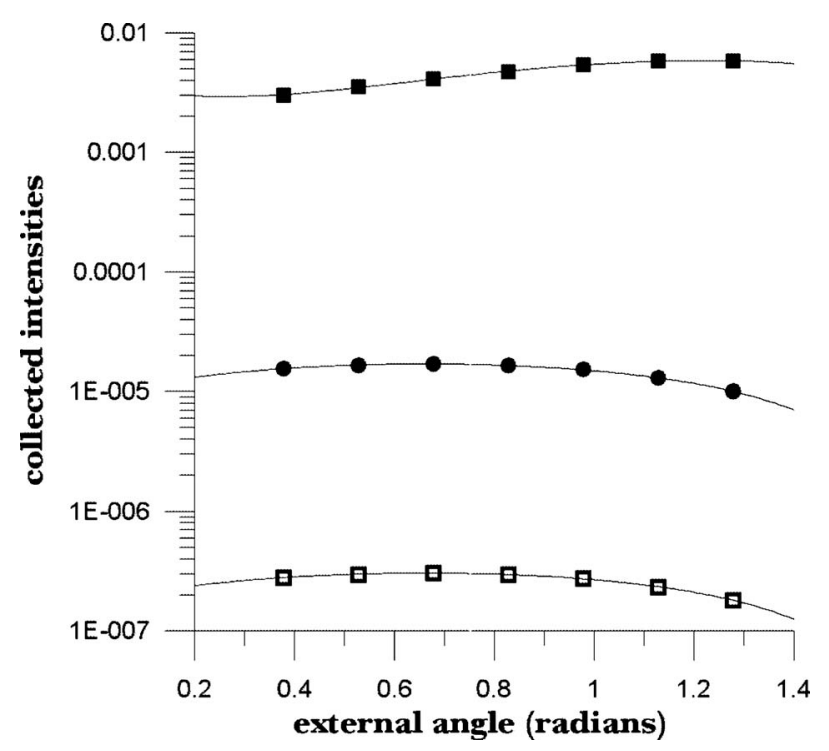

Fig. 8 Collected intensities as a function of angle between incoming beam and external normal, with distance between beam center and center of collection circle fixed at $100 \mu \mathrm{m}$. Solid squares=static tissue; solid circles=red blood cells; open squares=plasma.

red blood cells, and plasma, each the sum of contributions from the three layers. The value of $\theta^{\prime}$ was fixed at $53.2 \mathrm{deg}$ or $0.929 \mathrm{rad}$, which is the value used in our apparatus. (It was determined to be the best choice empirically, and the choice was confirmed by calculations of pressure-modulated intensities using our program, as explained later.) The optimal value of $d$ for tissue scattering is very small, since this scattering is dominated by the contribution of the top layer. From parabolic fits, we find that the optimum value of $d$ for scattering from red blood cells is $0.02030 \mathrm{~cm}$ and that for plasma is $0.02030 \mathrm{~cm}$. Getting more signal from greater depth ${ }^{23}$ requires larger values of $d$ for a given angle, since this maximizes the path length in layer $b$.

\section{Effects of Motion of Subsurface Tissues}

In many measurements, we do not obtain the separate contributions of the three phases to the scattering, but only the total intensity, which is dominated by the scattering of the top layer, consisting solely of static tissue. Information about the blood phases is obtained from modulated scattering intensities-i.e., we subtract the intensity measured for a pressed fingertip from the intensity measured for an unpressed fingertip. Pressure forces some blood and plasma out of layers $\mathrm{b}$ and $\mathrm{c}$ but has little effect on layer a. Therefore, the modulation or subtraction cancels out much of the large contribution of the static tissue. (However, as discussed in the following, because of the way pressure is applied in our experiments, the geometry of the skin surface is changedsee Fig. 3-and the cancellation is not complete.) Since we are interested in the scattering from red blood cells and plasma, we here establish the optimum geometric parameters for modulated scattering.

It is assumed that the pressure removes a fraction $f$ of the plasma and red blood cells in layers $\mathrm{b}$ and $\mathrm{c}$ and does not change the static tissue. Layer a is unaffected, but the volume

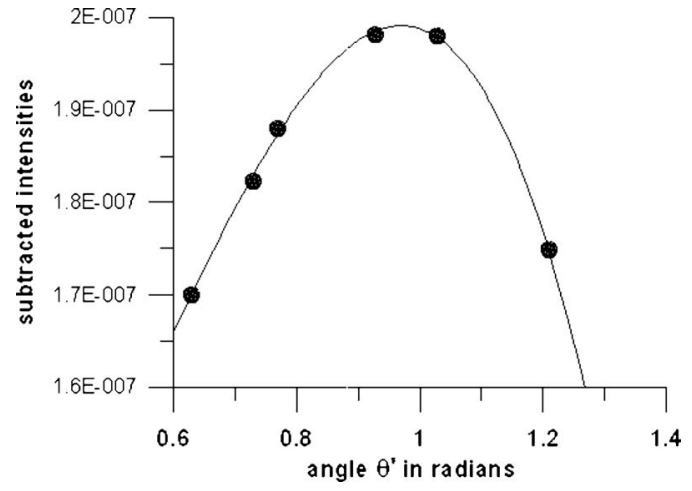

Fig. 9 Modulated (difference between unpressed and pressed) total intensities plotted against the angle $\theta^{\prime}$, with distance between center of beam and center of detector circle fixed at $200 \mu \mathrm{m}$. The curve in the figure is a cubic fit, for clarity. The optimum value of $\theta^{\prime}$ is $56.5 \mathrm{deg}$.

of layer b is decreased and, assuming no change in cross section, the thickness of layer $\mathrm{b}$ is decreased. (Since layer $\mathrm{c}$ is infinitely thick, no correction is required.) Considering unit volume, the volume of red blood cells is reduced by $f \phi_{r b}$ and the volume of plasma by $f \phi_{p b}$. The volume is multiplied by $1-f\left(\phi_{r b}+\phi_{p b}\right)$, so the new thickness is $t_{b} \times\left[1-f\left(\phi_{r b}\right.\right.$ $\left.\left.+\phi_{p b}\right)\right]$. Although static tissue is assumed not to be changed by pressure, its scattering will be changed because the screening of the incoming and outgoing light by blood is decreased, so the tissue scattering is not completely canceled out by the modulation or subtraction.

Figure 9 shows the subtracted intensities plotted against the angle $\theta^{\prime}$. For these calculations, $d$ was fixed at $0.02 \mathrm{~cm}$, and $f$ was 0.1 . The curve in the figure is a cubic fit, for clarity. From the data, we calculate the optimum value of $\theta^{\prime}$ to be $0.9783 \mathrm{rad}$ or $56.5 \mathrm{deg}$. This is very close to the value established experimentally earlier, $53 \mathrm{deg}$. We also conducted a series of calculations to establish the optimum value of $d$ for modulated intensities. Here, the angle $\theta^{\prime}$ was fixed at $0.98 \mathrm{rad}=56.1 \mathrm{deg}$, and $d$ was varied. For each value of $d$, intensities for unpressed and pressed tissue were calculated; these were subtracted to get the modulated intensities. The maximum occurs for $d=0.0203 \mathrm{~cm}$.

Another way to cancel out much of the scattering from static tissue is pulse modulation, in which the EE and IE are monitored over time. Each pulse gives an increase and then a decrease in both $\phi_{b}$ and $\phi_{p}$, the volume fractions of RBCs and plasma. Since the fluorescence per unit volume is relatively large for the RBCs, the IE increases when $\phi_{b}$ increases, regardless of what happens to $\phi_{p}$. This leads to only a small change in the $\mathrm{EE}$ originating from the RBCs themselves. However, their large scattering coefficient means that RBCs impede propagation of all light in the volume, so that an increase in $\phi_{b}$ increases the attenuation of the incoming and outgoing beams. Since static tissues have the largest volume fraction, increased $\phi_{b}$ decreases the total $\mathrm{EE}$, which explains why the IE and EE are found to be out of phase in pulsemodulated measurements. Our model can be used to calculate the effect.

We performed a series of calculations in planar geometry, with $\phi_{r b}+\phi_{p b}$ fixed at 0.008 , but $\phi_{r b}$ varying; $\phi_{r c}$ was always 


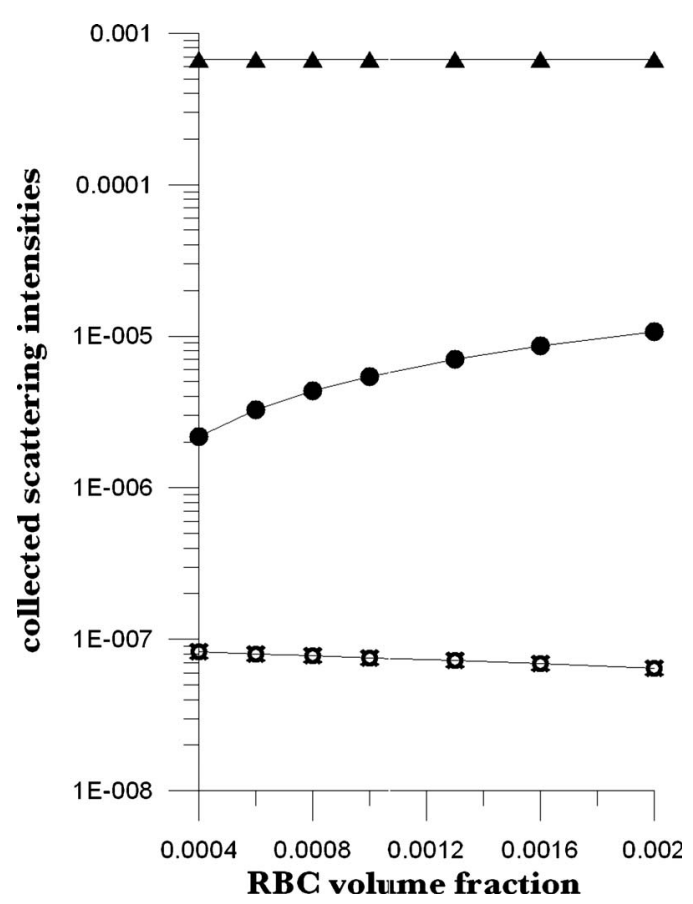

Fig. 10 Scattering intensities entering collection circle for static tissue (solid triangles), red blood cells (solid circles), and plasma (open squares), plotted against $\phi_{r b}$. The volume fraction of plasma in layer b in each case is $\phi_{p b}=0.0080-\phi_{r b}, \phi_{r c}=\phi_{r b} / 6$, and $\phi_{p c}=\phi_{p b} / 6$. Collected intensities for static tissue and for plasma decrease linearly with $\phi_{r b}$.

equal to $\phi_{r b} / 6$ and $\phi_{p c}$ equal to $\phi_{p b} / 6$. Note that $\phi_{r b} /\left(\phi_{r b}\right.$ $+\phi_{p b}$ ) is the hematocrit in layer b. The results (collected scattering intensity versus $\phi_{r b}$ ) are shown in Fig. 10. From top to bottom, the plots are for static tissue, RBCs, and plasma. The scattering from RBCs increases with $\phi_{r b}$, but not linearly, since the scattering attenuates both incoming and outgoing light. The scattering from the other two phases decreases linearly with $\phi_{r b}$. This explains why the IE and the EE change in opposite directions with pulse.

\section{Effects of Changes in Surface Topography: Doming and Ridges}

In our measurements, pressure modulation is done by pressing the fingertip against a metal plate in which a circular hole of diameter $D$ has been made, with the incoming laser beam and the detector on the other side of the plate. While pressing the fingertip in this way does remove fluids from the irradiated volume, it also extrudes tissue into the hole, resulting in formation of a dome. This changes the geometry of the experiment-in particular, the external angle $\theta^{\prime}$-which changes the internal angle $\theta$ and, possibly, the path lengths of the incoming and scattered radiation. The resulting change in the detected scattered radiation depends, obviously, on where the beam enters relative to the circumference of the hole. We now calculate the importance of this doming using our singlescattering model.

Figure 11 shows the geometry of the situation in the $x-z$ plane. The $x-y$ plane ( $x$ axis in the 2-D diagram) is the top surface of the skin with no doming, and the $z$ axis is in the
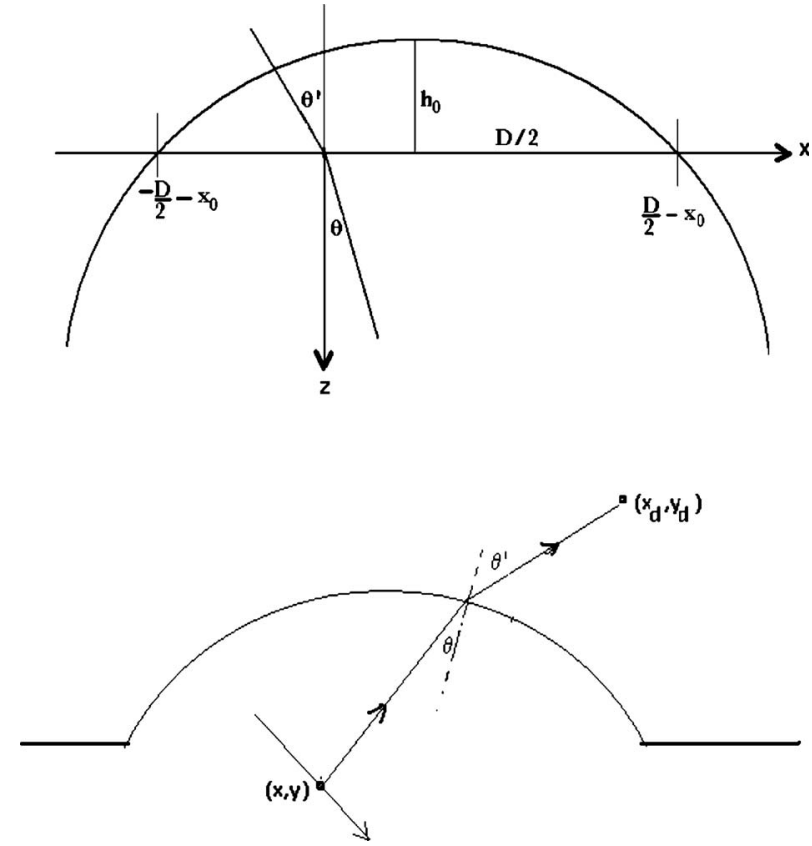

Fig. 11 Cross section of spherical dome formed by pressing fingertip into circular hole of diameter $D$, centered at $x=-x_{0}, y=0, z=0$. (a) From the maximum height of the dome above the $x-y$ plane, $h_{0}$, one can calculate the radius of the sphere, $R_{m}$. The angles $\theta^{\prime}$ and $\theta$ indicate the path that the beam would take in the absence of doming. (b) Path of beam of scattered radiation from $(x, y)$ to the center of the detector, $\left(x_{d}, y_{d}\right)$. The angles that the beam makes with the normal to the surface are $\theta$ (inside skin) and $\theta^{\prime}$ (outside skin), where $\theta$ and $\theta^{\prime}$ obey Snell's law.

downward direction, into the skin. The circular segment in Fig. 11(a) represents the dome formed by the fingertip's pressure on the plate; since it is defined by the hole in the plate, it has diameter $D$. We assume that the dome is spherical and call the maximum height above the $x$ - $y$ plane, at the dome center, $h_{0}$. In the $x-z$ plane, the dome extends from $x=-x_{0}$ to $x=D$ $-x_{0}$. The geometrical parameter to be varied is $x_{0}$, which corresponds to moving the measurement apparatus (laser beam and detector in unison, separated by a distance $d$ ) across the hole in the plate.

In the absence of doming, the incoming beam would cross the top surface of the skin (the $x-y$ plane) at the origin, and make an angle $\theta^{\prime}$ with the external normal (negative $z$ axis), as shown in the diagram. The angle it makes with the internal normal would be $\theta$. Clearly, the angle that the incoming beam makes with the external normal to the dome differs from $\theta^{\prime}$ [in Fig. 11(a), it is very close to 0], so its direction inside the skin differs from $\theta$; the difference is greater the farther one gets from the center of the dome.

We first calculate the radius of the dome, $R_{m}$, in terms of $D$ and $h_{0}$. Considering the continuation of $h_{0}$ to the center of the circle (sphere) of the dome, and a line from the center to the point $x=D / 2-x_{0}$, we find

$$
R_{m}=\left(h_{0}^{2}+\frac{D^{2}}{4}\right)\left(2 h_{0}\right)^{-1} .
$$

The equation of the circle is 


$$
\left(x-\frac{D}{2}+x_{0}\right)^{2}+\left(z-R_{m}+h_{0}\right)^{2}=R_{m}^{2} .
$$

Combining this with the equation for the incoming beam, $x=z \tan \theta^{\prime}$, we obtain the coordinates of the intersection point and the slope of the outward normal to the circle (spherical surface) at that point. Then, we calculate the angle the incoming beam makes with this normal and, using the Snell's law equation, we obtain $\theta$, the angle between the refracted beam inside the skin and the normal, as well as the transmission coefficient at the skin surface.

Then, as previously, we consider scattered intensity from every point along the trajectory of the beam in the skin. The intensity of scattering is proportional to the intensity of the beam, attenuated according to the path length from the entry point to the point of scattering. In Fig. $11(\mathrm{~b}),(x, y)$ is a point along the beam trajectory, and $\left(x_{d}, y_{d}\right)$ is the center of the detector. The normal to the sphere at the point where the scattered radiation crosses it is shown as a dashed line. The path that the scattered radiation takes to the detector is also altered because of the dome geometry, since refraction occurs at the skin surface (angles $\theta$ and $\theta^{\prime}$ relative to the normal to the surface). The point of intersection of the scattered beam with the surface must be calculated so that $\theta$ and $\theta^{\prime}$ obey Snell's law, and the transmission coefficient for the beam leaving the skin must be calculated.

Calculations of detected intensity were performed with $D$ $=0.200 \mathrm{~cm}, h_{0}=0.01 \mathrm{~cm}(100 \mu \mathrm{m}), d=0.020 \mathrm{~cm}$, and $\theta^{\prime}$ $=0.980$ (angle of incoming beam relative to the $z$ axis). The values of $D$ and $h_{0}$ are typical for our experimental apparatus and a finger with an unpressed diameter of $1 \mathrm{~cm}$. The beam source and the detector were assumed to be at $0.013 \mathrm{~cm}$ above the $z=0$ plane, with the center of the detector at $x_{d}$ and the center of the source beam at $x_{s}$, where $x_{d}-x_{s}=0.022 \mathrm{~cm}$. The range of meaningful $x_{s}$ values is from -0.12 to +0.08 ; the geometry makes $x_{0}$ (coordinate of the intersection of the beam center with the skin) greater by about 0.02 . For $x_{0}=-D / 2,0$, and $D / 2=0.100$, the angles that the beam inside the skin makes with the $z$ axis are $0.819,0.771$, and 0.706 , respectively; the corresponding transmission coefficients are 0.981 , 0.976 , and 0.963 . For $x_{0}$ approaching $-D / 2$ or $+D / 2$, we have to correct for the masking of the beam or the detector by the plate, since one or the other passes outside the aperture. Because of the nonzero widths of both the detector and the beam, the masking is gradual at each end. The contribution of reflected light from the aperture to either total EE or IE signal is discussed later.

Figure 12 shows how the calculated EE scattering intensities from static tissue (panel a), red blood cells (panel b), and plasma (panel c) change with $x_{s}$. Note that increasing $x_{s}$ corresponds to moving the aperture from right to left, or to moving the incoming beam and the detector from left to right, with the distance between the two fixed at $0.022 \mathrm{~cm}$. The masking of the beam is responsible for the drop of intensities to zero for $x_{s} \sim-0.12$ and the masking of the detector is responsible for the drop for $x_{s} \sim+0.08$.

Interestingly, the calculated EE intensity from static tissue first decreases (by almost 20\%) as $x_{s}$ increases until just before the centerline and then increases to slightly less than the original value at the other side of the aperture. In contrast, the

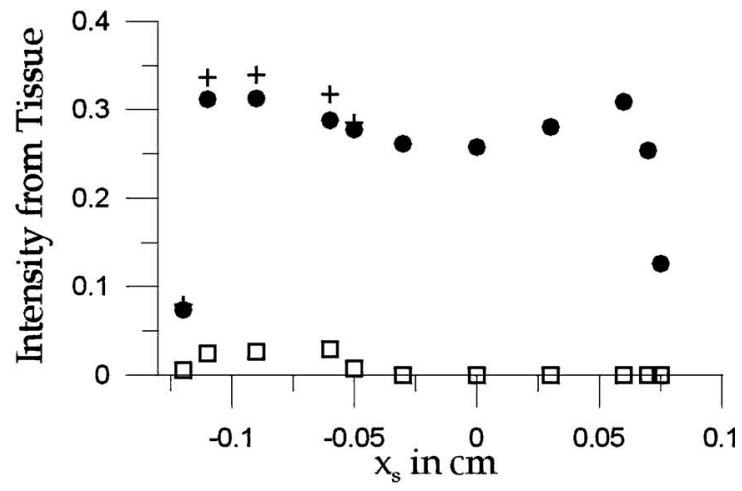

(a)

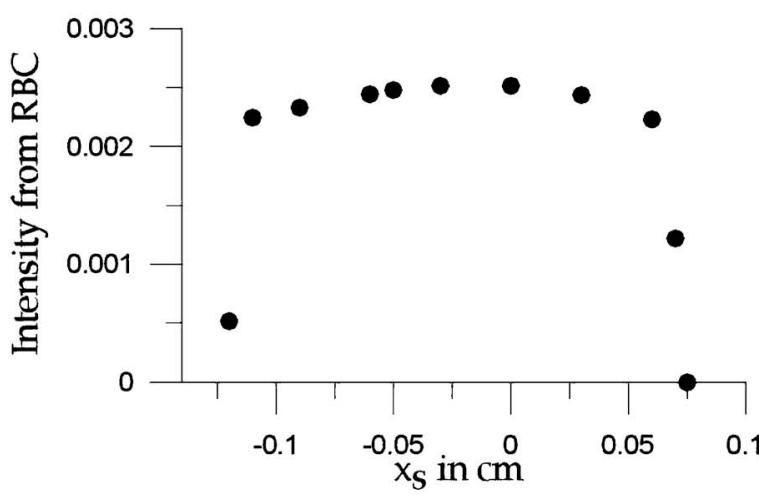

(b)

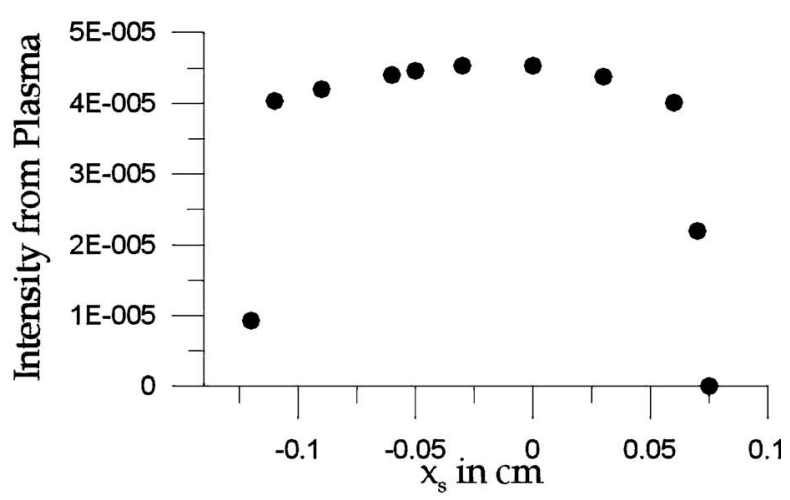

(c)

Fig. 12 Scattered intensity into detector as a function of position of beam source $x_{s}$, with detector centered at $x_{d}=x_{s}+0.022 \mathrm{~cm}$. Source and detector are at $0.013 \mathrm{~cm}$ above $z=0$. The beam enters at an angle relative to the normal of $0.980 \mathrm{rad}$. The aperture is at $z=0,-0.1 \mathrm{~cm}$ $\leqslant x \leqslant 0.1 \mathrm{~cm}$. Panels (a), (b), and (c) show scattered intensity from static tissue, red blood cells, and plasma. The open squares in panel (a) give the calculated intensity of specularly reflected light, and the crosses give the sum of the specular reflected light and the calculated intensity.

intensities from red blood cells and from plasma both go through maxima (each by about $10 \%$ total) as $x_{s}$ increases. Although the parameters used in the calculation are for the propagation and production of EE, this is exactly what is seen in the experimental results of Fig. 5 for the IE. Since these calculations deal with the tissue optics of the probing, any light that is produced in the same location as EE will have the same scanning behavior as EE. The opposite behaviors of 
scattering intensity from static tissue, which contributes to IE, and scattering intensity from blood, which contributes to EE, are also seen in Fig. 4. Here, the small intensity oscillations may be due to fingertip ridges, as discussed in more detail in the following, but their origin is the change in the incident angle of the beam.

Although the parameters associated with the production of $\mathrm{EE}$ are very different from those for the production of IE, the total IE production is dominated by the static tissue because of its dominant, $>95 \%$, volume fraction in any layer. In this connection, we have recently shown ${ }^{26}$ how the well-known "autofluorescence" of static tissue can be bleached by the excitation laser; this effect can be seen in the results in Fig. 5. We observe that the IE decays with time at each of the scan locations, unlike the EE. Thus, the calculated variation in production of EE reveals the location of the exciting light, which is available to be absorbed by whatever species cause the autofluorescence in static tissues, thereby also accounting for variation in the production of IE.

Unlike the measured IE, the measured EE contains a significant direct contribution from the light reflected specularly from the skin surface. The results of the calculations for the reflection from 1-cm-diam skin are shown in Fig. 12, panel a, as open squares. (The intensities are zero for $x_{s}>-0.03$ because the reflected light cannot enter the detector.) When added to the calculated EE, they produce the points labeled with crosses in Fig. 12(a). Visually scaled and offset, the results with and without the reflectance contribution are also shown as thick black lines in the EE and IE results in Fig. 5, respectively.

The actual shape of the dome relates to the size and turgor of the fingertip. Variation in the dome height and nonspherical dome shapes, consistent with a reasonable amount of turgor, shift, shape, and modulate the intensity of the specular contribution, accounting for the variation observed between individuals in Fig. 5. This can be seen in the decrease in EE as the displacement increases above $x_{s}=0$, i.e., $800 \mu \mathrm{m}$, compared to the calculations, which show a decrease after $x_{s}=0.8$, i.e., $1500 \mu \mathrm{m}$. In actual fingertips, the height decreases to zero more rapidly, approaching the aperture edge, than in a spherical cap. Also, the reflected light from the aperture itself (with associated chamfer, since the aperture is not infinitely thin) seen in the Delrin EE also contributes to the excess IE observed in vivo. Note that in general, the net IE signal is only $\approx 10 \%$ of the EE signal, and this excess fluorescence is much smaller for the Delrin, which has dome height 0 , so it cannot be easily seen in Fig. 5. We consider this an indirect effect of specularly reflected light.

It should be emphasized that the change in scattered intensity with $x_{s}$ (10 to $30 \%$; see Fig. 5) is much larger than the change in transmission coefficient $(2 \%)$. This confirms that the effect is mostly due to the changed beam direction inside the skin, which we have shown can lead to very large changes in scattered intensity. Of course, a small change in transmission coefficient near unity implies a large relative change in reflection coefficient, so that the specularly reflected light makes a substantial contribution to the observed EE. The different behaviors of static tissue scattering and blood scattering arise because the static tissue dominates the top layer, which does not contribute to the blood scattering. These observations

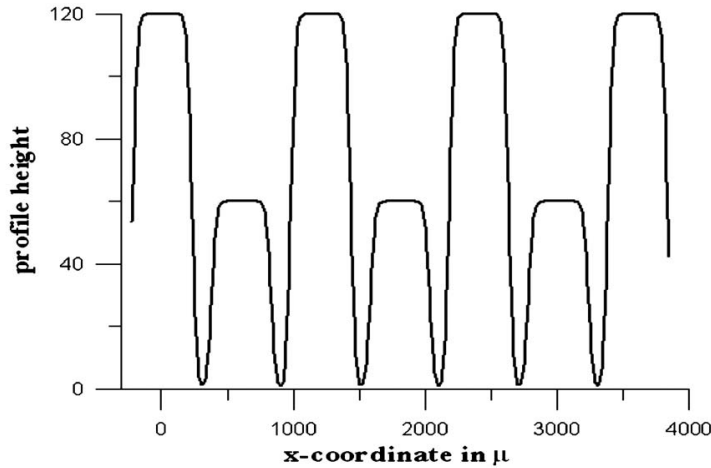

(a)

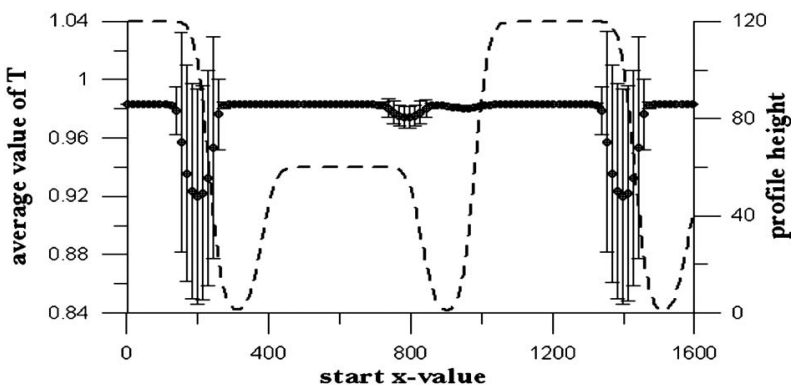

(b)

Fig. 13 (a) Profile representing fingertip ridges: double-Gaussian functions with spacing $600 \mu \mathrm{m}$, width $450 \mu \mathrm{m}$, shape $30 \mu \mathrm{m}$, and alternating heights of $120 \mu \mathrm{m}$ and $60 \mu \mathrm{m}$. (b) Calculated transmission coefficient averaged over width of beam ( $/$ and error bars) plotted against starting $x$ value for center of beam above profile (left scale), superposed on the profile.

are the basis for our discussion of the effect of fingertip ridges.

For a planar surface, the angle of incidence does not change with the placement of the beam. For a dome, it varies systematically with the position of the beam entry point. The actual surface of the skin is neither flat nor a dome because of the fingerprint ridges, which have a spacing of about $600 \mu \mathrm{m}$. Since the beam diameter is of the order $200 \mu \mathrm{m}$, the beam could illuminate mostly one ridge top, or straddle a ridge top and bottom. If the beam is incident near a ridge boundary, the angle of incidence of some of the incoming photons will be much larger or smaller than $53 \mathrm{deg}$, and we have shown that the scattering intensity can change significantly with a small change in the incident angle. Thus, moving the aperture across the fingertip produces not only a monotonic variation of intensity due to doming, but also oscillations commensurate with the spacing of the fingerprint ridges.

The profile shown in panel a of Fig. 13 is used to discuss this effect. The ridges are assumed to have a spacing of $600 \mu \mathrm{m}$; alternate ridges are assumed to have heights of $120 \mu \mathrm{m}$ and $60 \mu \mathrm{m}$. (The alternation in height is an observed feature of actual fingerprint ridges.) Each ridge is represented by the double-Gaussian function 


$$
y=\frac{h}{4}\left[1+\operatorname{erf}\left(\frac{x-x_{0}+\frac{w}{2}}{s \sqrt{2}}\right)\right]\left[1-\operatorname{erf}\left(\frac{x-x_{0}-\frac{w}{2}}{s \sqrt{2}}\right)\right],
$$

where $h$ is $120 \mu \mathrm{m}$ or $60 \mu \mathrm{m}, x_{0}$ is the center $(=7 \mu \mathrm{m}$, $607 \mu \mathrm{m}, 1207 \mu \mathrm{m}$, etc. $), w$ is the width $(450 \mu \mathrm{m})$, and $s$ is the shape parameter, equal to $30 \mu \mathrm{m}$. The values of the parameters are chosen to give the ridges and the valleys between them a shape close to what is actually observed. Starting at equally spaced values of $x(-222 \mu \mathrm{m},-207 \mu \mathrm{m},-192 \mu \mathrm{m}$, etc.) and height $164 \mu \mathrm{m}$, we calculate the trajectory of the incoming ray at $53 \mathrm{deg}$ to the vertical and determine where it intersects the profile and the angle $\theta^{\prime}$ it makes with the external normal to the profile at the point of intersection. From $\theta^{\prime}$, we obtain $\theta$ and calculate the transmission coefficient $T$, using Eq. (1).

Calculated transmittance $(T)$ varies widely as the entry point passes through a valley between ridges. Such large variations would be observed for infinitely narrow beams, but the beam has a width of $100 \mu \mathrm{m}$. Panel b shows the weighted average of transmittance over 7 points (symbol $\oplus$ ) and the population standard deviation (vertical bars), plotted against starting $x$ position. (Because the beam starts from above and is directed at $53 \mathrm{deg}$ from the vertical, the $x$ value of the entry point of the center of the beam is about $50 \mu \mathrm{m}$ to the right of the start value of $x$, the offset being different for different $x_{\text {start }}$. ) The profile is shown as a dashed line in panel $\mathrm{b}$. The averaging reduces the variation in transmittance to a few percent, but as we have seen, a small variation in transmittance is associated with a large variation in detected scattered intensity.

These calculated results explain what is observed in the IE and EE plots obtained by intentionally translating the fingertip while collecting CCD frames (Fig. 4). The IE and EE plots are complementary because the optimum angle of incidence is different for radiation scattered from static tissue, dominated by layer a, and for radiation scattered by blood, found only in layers $b$ and $c$. If one layer produces greater IE per unit volume than another, passing through the angle that optimizes signal from that layer will produce an increase in the observed IE. We chose the layer thicknesses to reflect the fact that the blood is mostly localized in a region between two others that barely contribute to the modulated signal when blood moves.

We considered the possibility that the oscillations in Fig. 4 are due to the variation in reflectance accompanying the variation in $T$. Using clean stainless steel ball bearings of different diameters to represent surfaces of different curvatures, we measured $^{27}$ the effect on the IE and EE of reflection from metal surfaces of various curvatures. The IE result is a small, featureless baseline shift over the entire collected spectrum. There is also an "inelastic" artifact because very highly off axis light can be generated using a ball bearing with very small radius of curvature and such highly antiparallel off axis light can be dispersed in the spectrograph across the entire CCD surface. However, opposite to what is observed in Fig. 4 , the purely reflective elastic and inelastic artifacts are in the same direction-i.e., collected light of both kinds is reduced.

\section{Discussion}

We have performed a series of calculations to help understand and represent (1) how incident and scattered light propagate in skin during an actual experiment and (2) how the IE and EE signals from the irradiated volume are affected by the measurement procedure. Even if one does not intend to perform a modulation experiment, the measurement process involves pressing the fingertip against an aperture in order to obtain reproducible registration with respect to the optical system, and the anatomical and physiological characteristics of skin in vivo require consideration of the effect of movement of subsurface materials, i.e., blood, on the measured signals.

We have discussed the situation in which ridged skin is probed by free-space coupled light, but many of our results are also relevant to experiments in which the incident light is coupled to a skin surface with different topography-e.g., forearm-using optical fiber. Although there might be no doming effect in this case, bringing fiber into registration with the skin surface inevitably indents the tissue with a concomitant movement of subsurface tissues. Also, all tissues have spatial texture on the scale of optical fiber diameters. Probing the nailbed vascular system that is accessible through the fingernail, ${ }^{28}$ as is done in commercial pulse oximetry, removes some soft tissue effects, but any pressure on the nail itself relative to the bone inside causes motion of suburface tissues.

With the assumptions and approximations of our model, we have predicted optimal values of the geometrical parameters of our instrument-i.e., incident angle and distance between the point of contact of the incident light with the skin surface and the collection circle. In fact, the signal was optimized using a procedure that we have presented in detail elsewhere, ${ }^{8,21}$ and the exact values of these and other parameters are close to those produced by the model. Probably, for best results, one should employ a real-time optical servo system to maintain certain optical metrics during the measurement process, as we already do ${ }^{8}$ to maintain mechanical consistency. Overall, the fact that we calculate reasonable values for the geometrical characteristics of the observed signals lends credibility to the entire modeling process.

Our model allows us to understand the effects of pressure modulation and ridged skin on the scattered intensity. The path of the laser light transmitted into the probed tissue is distorted by the surface topography, changing the angle of incidence and thereby the depth probed for a given experimental geometry. In an earlier small clinical study of noninvasive glucose measurement in vivo using combined fluorescence and Raman spectroscopy, ${ }^{4}$ we attempted to relate prediction errors to the measurement process. Production of fluorescence is much more efficient than production of Raman scattered light and so reflects the conditions at a greater depth. We calculate that Rayleigh/Mie scattered light originates from as deep as 2 millimeters, so observed fluorescence may actually approach that depth. Since fluorescence enters our algorithm for calculating glucose concentrations, the present study suggests that some of the prediction error may be associated with variation in the location of the incident laser relative to the fingerprint ridges. The results in Figs. 4 and 5 suggest a way to perform finger placement so as to achieve reproducible 
ridge placement with respect to the optical aperture and the incident laser.

We have presented a first principles-based model that is true to the experimental realities as well as the anatomy and physiology of the tissue. The skin is modeled as three-layer, and the requisite three phases are present: red blood cells, plasma, and static tissue, with the first two absent from the top layer. Values from the literature are used for the scattering and absorption coefficients and the volume fractions of the three phases. We assume that the phases are distributed randomly in the second and third layers, allowing calculation of average scattering and absorption coefficients of these layers. These determine the attenuation of incoming and scattered radiation, as well as the scattering probability at each point. By following the path of the light from the source outside the skin to the detector, we can calculate the intensity of detected radiation coming from each of the three phases [Eq. (5)]. The expression involves an integration over all points along the beam from which scattering may occur. To take into account that the laser beam is not infinitely thin, we average over an appropriate circular cross section.

Our calculations show that only a very small fraction of the collected light comes from layer c, due to the attenuation of both the incident and the scattered light. Because the production of wavelength-shifted light (IE) and the production of elastically produced light (EE) depend differently on the phases, we reproduce the different behaviors of the actual observed IE and EE. The optimum beam angle for EE from static tissue is much larger than for blood phases because the scattering from static tissue is dominated by the contribution of the top layer, which contains neither RBCs nor plasma. For blood and plasma scattering, a penetration into layers b and $c$ is required; for a fixed distance between beam and detector, this means a beam direction closer to the normal. Correspondingly, to observe more signal from greater depth requires larger values of $d$ for a given angle, since this maximizes the path length in layer $b$.

Our experiments do not measure the separate contributions of the three phases to the scattering, but obtain only the total intensity, which is dominated by the scattering of static tissue. To get information about the blood phases, we utilize tissue modulation-i.e., we subtract the measured total scattering intensity of a pressed fingertip from that of an unpressed fingertip. Since pressure forces some blood and plasma out of layers $\mathrm{b}$ and $\mathrm{c}$, but has little or no effect on layer a (as shown in Fig. 3), the modulation or subtraction cancels out much of the large contribution of the static tissue. Therefore, we established the optimum detector displacement for the modulated scattering, obtaining a value very close to the value found experimentally earlier-i.e., $\approx 53 \mathrm{deg}$ — giving us confidence in the model used. (We cannot align the optical system with greater precision than $\approx 2 \mathrm{deg}$.)

We have also tried to cancel out the scattering from static tissue by pulse modulation, since a pulse changes the volume fractions of RBCs, and we have previously published ${ }^{20}$ a "pulse-modulated" Raman spectrum of blood. Since the modulated IE is dominated by the contribution of RBCs, the inelastic scattering integral increases when the RBC volume fraction increases, allowing us to use the integrated IE to select a set of CCD frames of high RBC content and another set with low integrated fluorescence-i.e., IE- to subtract from the first. Using the IE to normalize these spectra as in previous studies, ${ }^{3}$ preliminary experiments show that a correlation exists between glucose concentrations obtained from these spectra and externally measured finger-stick blood glucose levels.

Most of our results show that elastic scattering intensities vary linearly with the various perturbations considered. We found a potentially important exception in a series of calculations in planar geometry, with $\phi_{r b}+\phi_{p b}$ fixed at 0.008 , but $\phi_{r b}$ varying; $\phi_{r c}$ was always equal to $\phi_{r b} / 6$ and $\phi_{p c}$ equal to $\phi_{p b} / 6$. As expected and as shown in Fig. 12, the scattering from RBCs increases with $\phi_{r b}$, and the scattering from the other two phases decreases linearly with $\phi_{r b}$. But the increase in scattering from RBCs is not a linear function of $\phi_{r b}$ because the elastic scattering by RBCs attenuates the scattered (outgoing) light, including that from the RBCs. These results explain why the observed inelastic and the elastic scattering integrals, IE and EE, change in opposite directions with the pulse. $^{5}$

In most experiments, we do not have a planar geometry because pressure applied to the fingertip for registration causes skin to extrude into the aperture, forming a dome. When the fingertip and the hole are moved relative to the laser beam and the detector, the scattering integrals IE and EE vary, because in the nonplanar geometry, the angle of incidence varies. When only the fingertip is moved, the point of incidence is at the same point of the dome, as long as the applied pressure is constant, and the angle of incidence is constant. However, there are oscillations corresponding to the fingerprint ridges (Fig. 4), with the inelastic and elastic scattering integrals changing in opposite directions. Calculations based on our model explain these effects.

In planar symmetry, the angle $\theta^{\prime}$ between the incoming beam and the external normal to the skin is independent of the point of entry of the beam. With a dome, the angle $\theta^{\prime}$ depends on the point of entry. We calculate the angle between the incoming beam and the external normal to the dome as a function of the position of the entry point of the beam and, using Snell's law, the angle between the refracted beam inside the skin and the internal normal, as well as the transmission coefficient at the skin surface. The transmission coefficient varies very little with the point of entry, but the variation in the reflection coefficient, itself about 0.02, is important because reflected radiation may contribute to the collected elastic scattering. The variation in detected scattered light intensity, however, is most important.

Figure 12 shows how the calculated elastic scattering intensities from static tissue, RBCs, and plasma change with beam entry position $x_{0}$ when the skin is stationary with respect to the aperture. The same calculated results are superimposed on the observed variations of intensities in Fig. 5 and are very well reproduced, especially when the direct and indirect effects of all the specularly reflected light are included. The (actual) intensity from static tissue decreases (by about $30 \%$ ) and then increases with $x_{0}$, while the intensities from red blood cells and from plasma increase (by about 10\%). The observed total EE intensity, dominated by the scattering from static tissue, decreases in the scanned direction faster than calculated, consistent with the notion that for a given dome height, the dome edges approach the edges of the aperture 
faster than would a spherical cap. The IE is characterized by very different scattering parameters from the EE, which compensates to some extent for the very different volume percentages of the phases. Nevertheless, the IE yield in Fig. 5 is close to that predicted for the $\mathrm{EE}$, suggesting that the IE from the plasma and RBCs is small compared to that from the static tissues. This is consistent with the observed ${ }^{5}$ net modulation of the time-dependent IE signal with the cardiac driven pulse, $\approx 5$ to $10 \%$.

Opposite variations in scattering intensity from static tissue, which dominates the IE, and scattering intensity from blood, which dominates the EE, are also seen in Fig. 4. In this case, the intensity oscillations are due to the changing incident angle of the beam as it passes across fingertip ridges. Since the spacing of the fingerprint ridges is about $600 \mu \mathrm{m}$, and the beam diameter is only $200 \mu \mathrm{m}$, the beam illuminates only a portion of a ridge, and the incident angle depends strongly on where on the ridge the beam impinges. The oscillations are in opposite directions because, as shown in Fig. 8, the optimum incident angles for static tissue and for blood are very different.

We need calculate only transmission coefficients for ridges, since the calculations for domed skin show that a small $(2 \%)$ change in transmission coefficient is associated with a large (10 to $30 \%$ ) change in scattered intensity. However, the observed variation is even greater, showing that more than one factor is in effect. By adjusting the parameters defining the surface geometry to get best fit to experimental results, one could gain information about skin surface topography. In the best case, a digital representation of the skin surface could be obtained by deconvolution of experimentally measured scattering intensity profiles.

The model shows the difference between the IE data obtained by moving the finger relative to the stationary aperture and optical system and the data obtained by scanning the aperture and the finger together across the optical system. The former, Fig. 4, corresponds to scanning a subsurface probe at constant depth, whereas in Fig. 5, the dome is probed at variable depth as the aperture is scanned across the optical system or, equivalently, the optical system scanned across the dome. Even with enough pressure to produce easily observed doming, the photographic study (Fig. 3) shows that the entry point of the laser moves less than $\approx 20 \mu \mathrm{m}$. The major axis of the elliptical spot is much wider than the motion of the spot induced by the increased dome height. The laser power for obtaining Fig. 3 was much reduced to obtain images with a minimum of CCD blooming. Thus, the model supports the idea that in a modulated experiment a nearly identical volume is probed for the pressed and unpressed states. According to Fig. 12, the signal collected during the unpressed measurement originates from deeper tissues. Because these are richer in blood than the more superficial tissues, the tissuemodulated-i.e., unpressed minus pressed-spectrum is enriched in Raman features originating with blood as originally claimed. ${ }^{3}$

This can be seen more clearly by reference to Fig. 5 in light of Fig. 12. During the unpressed phase of a tissue modulation cycle, the incident laser impinges on the skin surface near point $\mathrm{U}$, as marked on the IE graph. This is slightly to the side of the aperture center, indicated with $\mathrm{C}$, due to the slight doming caused by the small pressure used for registration.
Applying more pressure has two effects. First, the doming increases, and the point of contact of the laser moves farther from the centerline in the direction of the point labeled P. This decreases the IE and decreases the contribution of the static tissue to the net observed EE. Second, if a subsurface fluide.g., blood-moves out of the irradiated volume, the IE decreases even more.

The tissue-modulated spectrum obtained by subtracting the pressed from the unpressed spectrum therefore is enriched in Raman features and fluorescence originating from blood, in agreement with our earlier observations of correlation between the modulated Raman spectra and blood glucose levels measured independently. ${ }^{3}$ We stress that based on Fig. 3, the separation of the lines indicated by $\mathrm{U}$ and $\mathrm{P}$ is greatly exaggerated in Fig. 5 and that in reality they are $>95 \%$ superimposed on each other.

Although there are certainly other sources of variation in glucose concentrations calculated from in vivo Raman spectroscopy of volar-side fingertip capillaries, this study shows that one source is variation in the form of the dome, which could be mitigated by careful control of the measurement process. The calculations of Fig. 1 demonstrate that a singlescattering model should represent all qualitative aspects of probing real tissue in vivo by NIR radiation well. We have found that to be true, and with proper choice of the parameters, we could achieve quantitative agreement with observations. The radiation transfer approach is rigorous and very flexible and, when single scattering is assumed, a calculation requires only seconds, much less than multiple scattering models. $^{23}$

We have shown here that tissue modulation should produce spectral features originating with subsurface blood in the capillary network, making it possible to measure glucose and other blood analytes noninvasively using Raman spectroscopy. This approach has evolved so that, in addition to considering essential human factors of the measurement process, it strives to at least partially mitigate the effect of turbidity and intersubject skin differences, by managing RBC content and movement across test subjects and for one test subject across time.

We know of published data from two other Raman studies of noninvasive in vivo glucose measurement. ${ }^{29,30}$ It may be possible to further correct for turbidity effects ${ }^{31}$ using a data pretreatment based on an analytical treatment of multiple scattering effects. The proposed pretreatment would incorporate information from a reflectance spectrum, perhaps acquired simultaneously with the Raman data, to provide improved calibration across individuals. All of these measures must be explored to finally succeed in producing commercially viable devices that can meet the immense need for continuous noninvasive in vivo blood and tissue analysis.

\section{Conclusions}

The model presented herein for transmission and scattering of a laser beam through three layers of skin is able to interpret all our experimental observations. The model allows determination of the optimal configuration of laser beam and detector for collecting scattering from static tissue and blood phases. It can take into account the effect of pressure-induced deviations from a flat skin surface. It explains the origin of the alterna- 
tion between the IE and EE observed in both temporally modulated and spatially modulated experiments. The ability of this model to predict all of these aspects of the measurement process suggests that a single-scattering, isotropic model is adequate to describe the information derived from probing of skin using NIR radiation. Since the fingertip skin is highly vascularized and RBCs have by far the largest scattering coefficients of all the tissues involved, the single-scattering isotropic model should also suffice for modeling the probing of other skin locations with lower blood content, such as forearms. By affording an accurate interpretation of experimental observations as well as measurement artifacts, this model provides a firm theoretical basis for using the measured spectra of scattered light to determine, noninvasively and in vivo, blood properties like glucose concentration and hematocrit, as well as skin properties like turgor.

\section{Acknowledgments}

This research was supported by LighTouch Medical, Inc., and technical assistance in obtaining the data was provided by Dave Rice, John Fayos, and Dave Stehlik, all of Critical Link, LLC. Bin Deng, Ethan Voss, "Fast Eddie" Goodwin, Eric Lewis-Clark, and Dr. George Shaheen also assisted in data collection. The comments of the referees are appreciated.

\section{References}

1. A. Tu, Raman Spectroscopy in Biology: Principles and Applications, John Wiley and Sons, New York (1982).

2. R. L. McCreery, Raman Spectroscopy for Chemical Analysis, John Wiley \& Sons, New York (2000).

3. J. Chaiken, W. F. Finney, K. Peterson, C. M. Peterson, P. E. Knudson, R. S. Weinstock, and P. Lein, "Noninvasive, in vivo, tissue-modulated near-infrared vibrational spectroscopic study of mobile and static tissues: blood chemistry," Proc. SPIE 3918, 135-143 (2000).

4. J. Chaiken, W. F. Finney, P. E. Knudson, K. Peterson, C. M. Peterson, R. J. Bussjager, Y. Zhao, R. S. Weinstock, M. Khan, D. Hagrman, and P. Hagrman, "The effect of hemoglobin concentration variation on the accuracy and precision of glucose analysis using tissue modulated, noninvasive, in vivo Raman spectroscopy of human blood: a small clinical study," J. Biomed. Opt. 10, 031111 (2005).

5. J. Chaiken, J. Goodisman, B. Deng, R. J. Bussjager, and G. Shaheen, "Simultaneous, noninvasive observation of elastic scattering, fluorescence, and inelastic scattering as a monitor of blood flow and hematocrit in human fingertip capillary beds," J. Biomed. Opt. 14, 050505 (2009).

6. Sgt. D. Ashbaugh and R. C. M. P, "Quantitative-qualitative friction ridge analysis, an introduction to basic and advanced ridgeology," http://ridgesandfurrows.homestead.com/friction_skin.html (1999).

7. D. Hamm, "A review of "Morphogenesis of volar skin in the human fetus' by Alfred R. Hale," Identif. Can. 26, 1-3 (2003).

8. J. Chaiken, B. Deng, R. J. Bussjager, G. Shaheen, D. Rice, D. Stehlik, and J. Fayos, "Instrument for near-infrared emission spectroscopic probing of human fingertips in vivo," Rev. Sci. Instrum. 81, $034301(2010)$

9. L. Jones, "Perception and control of finger forces," in Proc. ASME Dynamic Systems and Control Division, R. J. Furness, Ed., ASME, DSC-vol. 64, pp. 133-137 (1998).

10. S. A. Mascaro and H. H. Asada, "Measurement of finger posture and three-axis fingertip touch force using fingernail sensors," IEEE Trans. Rob. Autom. 20, 26-35 (2004).

11. E. K. Chan, B. Sorg, D. Protsenko, M. O'Neil, M. Motamedi, and W. F. March, "Effects of compression on soft tissue optical properties," IEEE J. Sel. Top. Quantum Electron. 2, 943-950 (1996).
12. V. Tuchin, Tissue Optics, SPIE Press, Bellingham, WA (2007).

13. For one example, see R. Zhang, W. Verkruysse, B. Choi, J. A. Viator, B. Jung, L. O. Svaasand, G. Aguilar, and J. S. Nelson, "Determination of human skin optical properties from spectrophotometric measurements based on optimization by genetic algorithms," J. Biomed. Opt. 10, 024030 (2005).

14. S. L. Jacques, "Origins of tissue optical properties in the UVA, visible, and NIR regions," in OSA TOPS on Advances in Optical Imaging and Photon Migration, R. R. Alfano and J. G. Fujimoto, Eds., vol. 2, pp. 364-369, Optical Society of America, Washington, D.C. (1996).

15. A. M. K. Enejder, T. G. Scecina, J. Oh, M. Hunter, W. C. Shih, S. Sasic, G. Horowitz, and M. S. Feld, "Raman spectroscopy for noninvasive glucose measurements," J. Biomed. Opt. 10, 031114 (2005).

16. P. J. Caspers, G. W. Lucasesen, and G. J. Puppels, "Combined in vivo confocal Raman spectroscopy and confocal microscopy of human skin," Biophys. J. 85, 572-580 (2003).

17. Rough estimate also based on capillary dimension data in P. Williams, Ed., Gray's Anatomy, 38th ed., p. 1465, Churchill Livingston, New York (1999), combined with loop-width data in C. Lentner, Ed., Geigy Tables, 8th ed., vol. 5, p. 545, CIBA-Geigy, Basel (1990).

18. T. F. McAnish, Ed., Physics in Medicine and Biology Encyclopedia, vol. 1, p. 105, Pergamon Press, New York (1986).

19. E. Salomatina, B. Jiang, J. Novak, and A. N. Yaroslavsky, "Optical properties of normal and cancerous human skin in the visible and near infrared spectral range," J. Biomed. Opt. 11, 064026 (2006).

20. M. Meinke, G. Muller, J. Helfmann, and M. Friebel, "Optical properties of platelets and blood plasma and their influence of the optical behavior of whole blood in the visible and near infrared wavelength range," J. Biomed. Opt. 12, 014024 (2007).

21. J. Chaiken, K. Ellis, P. Eslick, L. Piacente, and E. Voss, "Noninvasive in vivo tissue and pulse modulated Raman spectroscopy of human capillary blood and plasma," Proc. SPIE 6093, 609305 (2006).

22. S. A. Mascaro and H. H. Asada, "The common patterns of blood perfusion in the fingernail bed subject to fingertip touch force and finger posture," Haptics-e 4(3), http://www.haptics-e.org (2006).

23. P. Matousek, M. D. Morris, N. Everall, I. P. Clark, M. Towrie, E. Draper, A. Goodship, and A. W. Parker, "Numerical simulations of subsurface probing in diffusely scattering media using spatially offset Raman spectroscopy," Appl. Spectrosc. 59, 1485-1492 (2005).

24. M. Born and E. Wolf, Principles of Optics, 6th ed., p. 40, Pergamon Press, New York (1980).

25. A. H. Stroud and D. Secrest, Gaussian Quadrature Formulas, Prentice-Hall, Englewood Cliffs, NJ (1966).

26. B. Deng, C. Wright, E. Lewis-Clark, G. Shaheen, R. Geier, and J. Chaiken, "Direct noninvasive observation of near-infrared photobleaching of autofluorescence in human volar side fingertips in vivo," Proc. SPIE 7560, 75600P-1 (2010).

27. J. Chaiken, E. Voss, R. J. Bussjager, and G. Shaheen, "Towards an improved assignment of spectral features in tissue modulated noninvasive Raman spectroscopy of human fingertips," Proc. SPIE 6430, 643004 (2007).

28. J. Chaiken, W. F. Finney, X. Yang, P. E. Knudson, K. Peterson, C. M. Peterson, R. S. Weinstock, and D. Hagerman, "Progress in the noninvasive, in vivo, tissue-modulated Raman spectroscopy of human blood," Proc. SPIE 4254, 216-227 (2001).

29. I. Barman, G. P. Singh, R. R. Dasari, and M. S. Feld, "Turbiditycorrected Raman spectroscopy for blood analyte detection," Anal. Chem. 81, 4233-4240 (2009).

30. J. Lipson, J. Berhardt, U. Block, W. R. Freeman, R. Hofmeister, M. Hristakeva, T. Lenosky, R. McNamara, D. Petrasek, D. Veltkamp, and S. Waydo, "Requirements for calibration in noninvasive monitoring by Raman spectroscopy," J. Diabetes Sci. Technol. 3, 233-241 (2009).

31. Another recent addition to the discussion of turbidity effects in Raman spectroscopy is R. Steponavicius and S. N. Thennadil, "Extraction of chemical information of suspensions using radiative transfer theory to remove multiple scattering effects: application to a model two-component system," Anal. Chem. 81, 7713-7723 (2009). 